\title{
Musical Hallucination Caused by Ceftazidime in a Woman with a Hearing Impairment
}

\author{
Chan II Song ${ }^{1}$, Young-Eun Jung ${ }^{2}$ \\ ${ }^{1}$ Department of Otorhinolaryngology, Yonsei University College of Medicine, Seoul, ${ }^{2}$ Department of Psychiatry, Jeju National University School \\ of Medicine, Jeju, Korea
}

\begin{abstract}
Musical hallucinations remain a poorly understood clinical phenomenon, possibly because these types of hallucination have multiple causes and are rarely the focus of published reports. Here, the case of a 51-year-old female patient with a hearing impairment who developed musical hallucinations during treatment with ceftazidime, a third-generation cephalosporin, is presented. She responded to the discontinuation of ceftazidime and the initiation of low-dose olanzapine treatment. Musical hallucinations associated with ceftazidime are very rare, and the mechanisms underlying its occurrence remain unknown. Further studies will be necessary to determine the pathophysiology of adverse psychiatric reactions associated with ceftazidime.
\end{abstract}

KEY WORDS: Ceftazidime; Musical hallucination; Adverse drug reaction; Hearing impairment.

\section{INTRODUCTION}

Musical hallucinations, which are also known as musical hallucinosis or musical-ear syndrome, are characterized by songs, tunes, harmonics, rhythms, and/or timbers. ${ }^{1)}$ The exact prevalence of musical hallucinations is unknown, although estimates as high as $2.5 \%$ have been reported in older patients with hearing impairments. ${ }^{2)}$ Various types of dysfunction within an extensive network of interconnected brain areas are thought to be the potential causes of musical hallucinations, but most cases are likely due to multifactorial etiologies. The etiology of musical hallucinations can be divided into five groups: secondary to auditory deprivation, focal cerebral lesions, psychiatric pathology, epilepsy, and secondary to pharmaceuticals or metabotoxic causes. ${ }^{3-7)}$

Here, the case of a patient with no personal or family history of psychiatric disorders who complained of musi-

Received: July 10, 2017 / Revised: July 31, 2017

Accepted: August 1, 2017

Address for correspondence: Young-Eun Jung, MD, PhD Department of Psychiatry, Jeju National University School of Medicine, 15 Aran 13-gil, Jeju 63241, Korea

Tel: +82-64-717-1850, Fax: +82-64-717-1849

E-mail: jyejye77@daum.net

ORCID: https://orcid.org/0000-0001-7608-0009 cal hallucinations associated with the administration of ceftazidime is presented. Ceftazidime is a third-generation cephalosporin that belongs to a group of beta-lactam antibiotics used for the treatment of severe infections, particularly ciprofloxacin-resistant Pseudomonas aeruginosa infections. Ceftazidime is associated with several adverse reactions within the central nervous system, including headaches, dizziness, convulsions, manic episodes, and hallucinations. ${ }^{8-12)}$ To our knowledge, this is the first report of musical hallucinations associated with ceftazidime in the absence of other neurological or psychiatric symptoms.

\section{CASE}

A 51-year-old woman presented for a consultation for bilateral otorrhea at our otorhinolaryngology department. The patient had tympanic membrane perforations and active pus discharge in both ears; a bacterial culture of the otorrhea samples showed ciprofloxacin-resistant $P$. aeruginosa. Computed tomography (CT) scans of the temporal bone revealed chronic otitis media in both ears. Although the patient had hearing impairments in both ears, it was possible to appropriately communicate with her because she had speech discrimination scores of $96 \%$

(c) This is an Open-Access article distributed under the terms of the Creative Commons Attribution Non-Commercial License (http://creativecommons.org/licenses/by-nc/4.0) which permits unrestricted non-commercial use, distribution, and reproduction in any medium, provided the original work is properly cited. 
for both ears without hearing aids on a speech audiometry exam. Her condition was diagnosed as bilateral chronic otitis media; thus, surgery and administration of antibacterial agents were recommended as treatment. However, the patient did not want to undergo surgery and was treated only with intravenous administrations of ceftazidime ( $2 \mathrm{~g} /$ day), which is a third-generation cephalosporin effective against ciprofloxacin-resistant $P$. aeruginosa. The patient complained of auditory hallucinations that began 1 to 2 days after administration of ceftazidime. She had suddenly begun hearing songs with lyrics that repeated themselves indefinitely before changing into different pieces of music. In the beginning, the music was soft and did not interfere with her daily life, but the loudness of the music eventually increased, and the musical hallucinations were present from the moment she awoke until late at night.

The patient was thoroughly assessed, but neurological examinations and a brain CT scan revealed no significant abnormalities. Blood examinations, including a hemogram and liver function, kidney function, serum electrolyte, and thyroid function tests, were all within normal limits; there was no individual or family history of any psychiatric or medical illness in the index patient, and no history of substance abuse was present. A psychiatric interview and examination were unable to identify any factors responsible for the musical hallucinations other than ceftazidime. Thus, administration of ceftazidime was discontinued after 2 weeks. However, the musical hallucinations persisted, and the patient began treatment with olanzapine tablets ( $2.5 \mathrm{mg} /$ day). During subsequent follow-up visits, the sounds decreased in intensity and did not interfere with her daily life, but were not completely resolved. As a result, the olanzapine dose was increased to 5 $\mathrm{mg} /$ day; but, due to increased sedation, it had to be decreased to $2.5 \mathrm{mg} /$ day again. The patient was followed for approximately 6 months and she maintained improvements.

\section{DISCUSSION}

Musical hallucinations are associated with being female, hearing impairments, brain diseases affecting the non-dominant hemisphere, temporal lobe lesions, and mental disorders such as depression, schizophrenia, and obsessive-compulsive disorder. ${ }^{1)}$ In the present case, the musical hallucinations were associated with moderately severe-to-severe hearing loss in the absence of other neuropsychiatric conditions. Keshavan and Schooler ${ }^{13)}$ suggested that musical hallucinations derive from memory tracts, which is a concept they refer to as "parasitic memory." It has also been suggested that musical hallucinations are the result of sensory deprivation and may be similar to the effects of sensory deprivation in patients with phantom limbs. ${ }^{14)}$

Previous studies have reported occurrences of ceftazidime-induced encephalopathy with or without hallucinations. ${ }^{9,10,15)}$ The central nervous system effects associated with ceftazidime manifest primarily in elderly patients (over 60 years of age) and in patients with impaired renal function in whom the dose of ceftazidime is not appropriately adjusted. Because ceftazidime is not metabolized and is exclusively excreted by the kidney, great care should be exercised when administering ceftazidime to patients with renal disease. ${ }^{15)}$ A previous report described the case of a 62-year-old woman with auditory and visual hallucinations in the absence of other psychiatric symptoms. The occurrence of hallucinations in this elderly patient was presumed to be due to the prolonged half-life of the drug attributable to impaired renal clearance. ${ }^{8)}$ Compared to that case, the present patient was relatively young and did not have a renal disease. Jackson and Berkovic ${ }^{10)}$ suggested that ceftazidime toxicity may result in disturbances to deep midline gray matter which, in turn, cause transient toxic peduncular hallucinosis. The patient in that case experienced vivid visual and auditory hallucinations that closely resembled the nature of peduncular hallucinosis, which is usually associated with lesions involving the deep midline gray matter of the midbrain, hypothalamus, or thalamus. Their hypothesis may not be applicable to the present case of persistent musical hallucinations that likely arose from other underlying mechanisms. However, it was impossible to determine whether the patient had any anatomical or functional lesions because she refused further evaluations with magnetic resonance imaging or an electroencephalogram.

The persistent musical hallucinations in the present patient may be attributed to delays in identifying the adverse drug reaction to ceftazidime. Although the musical hallucinations began just 1 or 2 days after administration of ceftazidime, the possible cause of the symptoms was not 
identified for 2 weeks, and administration of ceftazidime was continued during this time. Previous case reports determined that when ceftazidime administration was discontinued immediately after symptoms began, hallucinations subsided after several days. ${ }^{8-10)}$

General recommendations for the treatment of musical hallucinations have yet to be established, but a variety of therapeutic options have been tried in case reports and small case series. ${ }^{1)}$ Treatments are aimed the underlying cause, if it is known, but not all patients require treatment, as musical hallucinations may be self-limiting and reassurance is often sufficient in patients with low levels of distress. ${ }^{1)}$ All pharmacological treatments for idiopathic musical hallucinations are off-label, and it has been shown that some antipsychotics or acetylcholinesterase inhibitors have an effect on musical hallucinations. ${ }^{1,16,17)}$

In conclusion, it is emphasized that although musical hallucinations are very rare, clinicians should try to evaluate their existence and consider multiple pathologies. The majority of cases in which treatment has been effective depended on the resolution of the underlying cause such as suspending the responsible pharmaceuticals. However, treating musical hallucinations remains a challenge as there is currently no curative treatment. Further studies will be necessary to determine the pathophysiology, natural course, and treatment results of musical hallucinations.

\section{REFERENCES}

1. Coebergh JA, Lauw RF, Bots R, Sommer IE, Blom JD. Musical hallucinations: review of treatment effects. Front Psychol 2015;6:814.

2. Cole MG, Dowson L, Dendukuri N, Belzile E. The prevalence and phenomenology of auditory hallucinations among elderly subjects attending an audiology clinic. Int I Geriatr Psychiatry 2002;17:444-452.

3. Sanchez TG, Rocha SC, Knobel KA, Kii MA, Santos RM,
Pereira CB. Musical hallucination associated with hearing loss. Arq Neuropsiquiatr 2011;69:395-400.

4. Golden EC, Josephs KA. Minds on replay: musical hallucinations and their relationship to neurological disease. Brain 2015;138:3793-3802.

5. Schielke E, Reuter U, Hoffmann O, Weber JR. Musical hallucinations with dorsal pontine lesions. Neurology 2000;55: 454-455.

6. Evers S. Musical hallucinations. Curr Psychiatry Rep 2006;8: 205-210.

7. Tomar A, Cheung G. Musical hallucinations induced by drugs. Int Psychogeriatr 2007;19:1169-1172.

8. al-Zahawi MF, Sprott MS, Hendrick DJ. Hallucinations in association with ceftazidime. BMJ 1988;297:858.

9. Douglas MA, Quandt CM, Stanley DA. Ceftazidime-induced encephalopathy in a patient with renal impairment. Arch Neurol 1988:45:936-937.

10. Jackson GD, Berkovic SF. Ceftazidime encephalopathy: absence status and toxic hallucinations. I Neurol Neurosurg Psychiatry 1992; 55:333-334.

11. Quandt-Herrera P, Suarez-Jesus J, Yelmo-Cruz S. Antibiomania: Secondary mania associated with ceftazidime. I Clin Psychopharmacol 2015;35:619-621.

12. Slaker RA, Danielson B. Neurotoxicity associated with ceftazidime therapy in geriatric patients with renal dysfunction. Pharmacotherapy 1991;11:351-352.

13. Keshavan MS, Schooler NR. First-episode studies in schizophrenia: criteria and characterization. Schizophr Bull 1992; 18:491-513.

14. Auffarth IS, Kropp S. Musical hallucination in a patient after cochlear implantation. I Neuropsychiatry Clin Neurosci 2009;21:230-231.

15. Joseph J, Vimala A. Ceftazidime-induced myoclonus and encephalopathy in hemodialysis patient. Indian J Nephrol 2015; 25:61-62.

16. Blom JD, Coebergh JA, Lauw R, Sommer IE. Musical hallucinations treated with acety/cholinesterase inhibitors. Front Psychiatry 2015;6:46.

17. Mansoor D, Ganzini L. Musical hallucinations successfully treated with antipsychotic medications: three case reports. Psychosomatics 2014;55:191-193. 